\title{
The role of cancer-associated fibroblasts in esophageal cancer
}

\author{
Jiangfeng Wang, Guangyu Zhang, Jianbo Wang, Lu Wang, Xiaochen Huang and Yufeng Cheng*
}

\begin{abstract}
Fibroblasts are known as critical stromal cells in wound healing by synthesizing extracellular matrix and collagen. A subpopulation of them is called cancer-associated fibroblasts (CAFs), because their production of proteins participated in various biological activities including tumor cell proliferation, invasion and metastasis. Currently some studies shed light on their role in esophageal cancer which was an aggressive cancer with a dismal survival and high rate of metastasis. Thus, to find cures for it relies on elucidating the epithelial-fibroblasts crosstalk. Herein, we reviewed the present knowledge of the CAFs' role in esophageal premalignant condition, cancer initiation, progression, metastasis and prognosis prediction and further provided some insights into its clinical application.
\end{abstract}

Keywords: Esophageal cancer, Cancer-associated fibroblasts, Carcinogenesis, Progression, Metastasis, Prognosis, Treatment

\section{Background}

More than 450,000 people worldwide are inflicted with esophageal cancer featuring early metastatic spread and poor prognosis every year, whose overall 5 -year survival ranged between 15 and $25 \%$ [1]. The poor outcomes are not only related to the tumor-cell but also impacted by the stromal fibroblasts [2]. Previously, the investigations of fibroblasts usually focused on wounds healing. Activated fibroblasts with high-proliferation and high-secretion phenotype generate extracellular matrix (ECM) and regulate inflammation. Once the wounds are healed, the number of activated fibroblasts is greatly reduced and the quiescent fibroblasts are restored [3]. Currently, some studies concentrated on the role of perpetually activated fibroblasts in the microenvironment of various types of cancers including esophageal cancer $[3,4]$. And these activated fibroblasts are known as cancer-associated fibroblasts (CAFs).

It becomes clear that CAFs are prominent regulators involved in the entire course of cancer development. They provide esophageal cancer cells with a suitable environment for carcinogenesis, proliferation, angiogenesis and

\footnotetext{
*Correspondence: qiluflk@163.com

Department of Radiation Oncology, Qilu Hospital of Shandong University, No 107 West Wenhua Road, Jinan 250012, People's Republic of China
}

invasion via secreting factors $[2,5-10]$. Thus, the aims of the study were to summarize CAFs' role in esophageal cancer and to provide some insights into its future clinical application.

\section{The origins of CAFs}

To better understand the impact of CAFs on esophageal cancer development, the first step is to know their diverse origins, which included normal fibroblasts (NFs), bone marrow-derived cells (BMDCs), endothelial cells, hematopoietic stem cells (HSCs), epithelial cells, pericytes and adipocytes [11] (Fig. 1).

NFs in esophageal cancer were reported to be a source of CAFs in response to the paracrine of cancer cells. Nouraee et al. [12] co-cultured NFs with esophageal carcinoma cells and observed CAF markers were upregulated. It was suggested that some microRNAs may got involved in this process. The transformation may be attributed to the elevated release of microRNA-21 from esophageal squamous cell carcinoma cells (KYSE-30). In addition, microRNA-27 was also observed to manipulate the transformation of NFs into CAFs [13]. Transforming growth factor beta (TGF $\beta$ ) also was promoter of this process [14]. BMDCs were another source of esophageal activated fibroblasts. Hutchinson et al. [15] transplanted mice with bone marrow expressing beta galactosidase. 


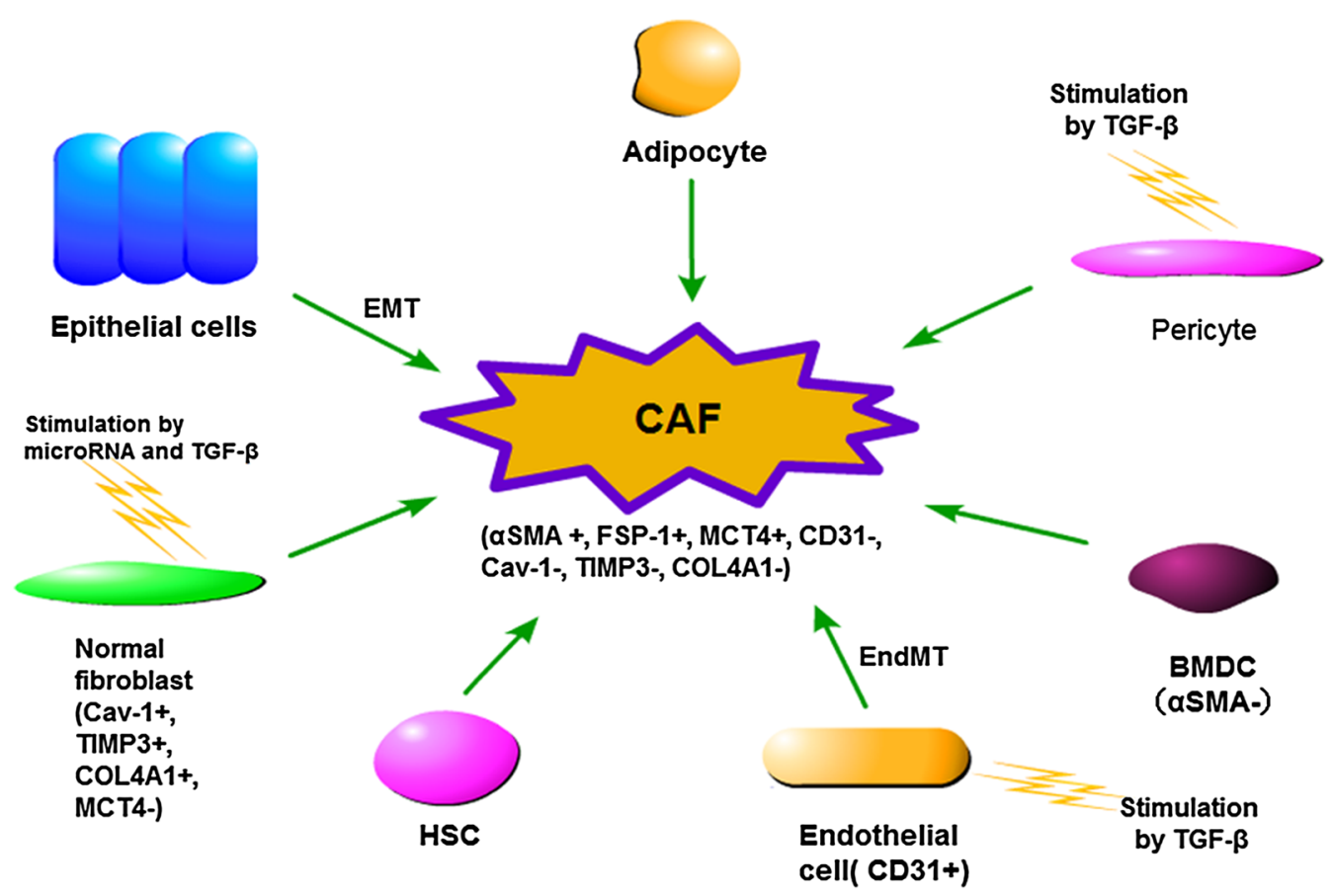

Fig. 1 Origin of CAFs. CAFs can derive from a variety of cells, including normal fibroblasts (NFs), bone marrow-derived cells (BMDCs), endothelial cells, hematopoietic stem cells (HSCs), epithelial cells, pericytes and adipocytes. Previerous studies showed their nature to transform to CAF phenotype. TGF- $\beta$ and microRNAs were reported as the drivers of this transformation

Then the mice suffered Barrett's metaplasia, in which authors found activated fibroblasts labeled with not only their specific marker $\alpha$ smooth muscle actin (SMA), but also beta galactosidase. It implicated they were derived from BMDCs. In vivo, authors found BMDCs-derived CAFs in a patient with esophageal adenocarcinoma (EAC) [15]. In this research, a male patient was diagnosed as EAC 10 years after bone marrow transplantation from a female donor. Through X/Y fluorescent in situ hybridization and anti- $\alpha$-SMA staining, they discovered $12.57 \%$ CAFs were originated from the donor bone marrow [15]. However, the small sample size was the limitation of this study. It should be verified in more patients. In other cancers, endothelial cells, HSCs, epithelial cells, pericytes and adipocytes were proved as CAFs resources [11, 16-20], but they remained unclear in esophageal cancer, which needed further researches.

\section{The role of fibroblasts in esophageal premalignant condition}

Reflux esophagitis is stimulated by gastric acid and bile reflux. Chronic exposure to gastroesophageal reflux will transform esophageal squamous epithelium to columnar epithelium, which is known as Barrett's esophagus (BE). It is the precancerous lesion of esophageal cancer [21]. Therefore, in order to investigate the whole course of cancer development, some studies focused on the relationship between fibroblasts and precancerous disease.

Interleukin (IL) $-1 \beta$ and IL- 6 were found in mucosal specimens of reflux esophagitis patients. In the study of Rieder et al. [22], treatment of IL-1 $\beta$ on human esophageal fibroblasts induced increased production of IL-6 which was the critical proinflammatory cytokines.

BE metaplasia could be induced by fibroblasts-derived factors. In esophageal mucosal biopsy specimens from reflux esophagitis patients, Farzana et al. [5] found that heparin-binding EGF-like factor (HB-EGF) was robustly expressed in fibroblasts. Additionally, in vitro stimulation of normal esophageal squamous epithelial cell with HB-EGF enhanced expression of the malignant markers including $\mathrm{Cdx} 2$, cytokeratin 7 and villin. $\mathrm{Cdx} 2$ was involved in intestinal metaplasia [23, 24]. Cytokeratin 7 and villin were columnar cells markers. Proliferation of BE was also promoted by fibroblasts. In Barrett's esophageal fibroblast culture, the crucial enzyme of prostaglandins E2 (PGE2) cyclooxygenase (COX)-2 was detected. Adding COX-2 inhibitor into epithelial cell cultures suppressed its proliferation, while PGE2 treatment rescued the Barrett's esophageal epithelial cell proliferation [25]. Taken together, proteins from fibroblasts were responsible for BE's inducement and proliferation. 


\section{The role of CAFs in esophageal carcinogenesis}

Esophageal squamous cell carcinoma (ESCC) initiation is commonly attributed to hot drinks and alcohol intake [26]. Whereas, EAC carcinogenesis is reported to closely correlate to BE [25]. Many factors participating in carcinogenesis were investigated. And CAFs was just one of them.

In ESCC tissues, transforming growth factor $\beta 1$ (TGF $\beta 1$ ) and hepatocyte growth factor (HGF) were stained in fibroblasts. Their levels were increased gradually from low-and high-grade intraepithelial neoplasia to carcinoma in situ and ESCC groups [27]. So fibroblasts were speculated to promote carcinogenesis of ESCC through TGF $\beta 1$ and HGF. However, no further studies investigated the mechanism.

Inflammatory factors produced by CAFs got involved in ESCC carcinogenesis as well. Achyut et al. [28] deleted TGF $\beta$ receptor 2 (T $\beta R I I)$ stromal fibroblasts in the mice forestomach. As a result, adjacent epithelial cells showed little expression of cyclin-dependent kinase inhibitor (CKI), high-level COX2, increased proliferation and decreased apoptosis. Then, in human ESCC specimens, they found that TRRII was downregulated in CAFs, inflammatory factors COX2 and DNA damaging indicators were enhanced and CKIs were decreased or even lost. Because squamous cell carcinoma of mouse forestomach was similar to human ESCC, so authors assumed CAFs of ESCC led to DNA damage, unlimited cell proliferation and malignant transformation. However, the limitation of the research is obvious. The cell source of mouse fore stomach led to faint conclusion, which needed robust investigation in ESCC cells.

Verbeek et al. [29] reported that CAF-derived Tolllike receptor-4 (TLR-4) (an innate immune system activator) was involved in the progression from $\mathrm{BE}$ to EAC. Expression of TLR-4 progressively increased from reflux esophagitis patients, patients with $\mathrm{BE}$, to those with EAC. It was found to boost COX-2 level in $\mathrm{BE}$, which was critical in EAC carcinogenesis, because it owned anti-apoptotic potential in vitro [30] and its inhibitor had ability to reduce incidence of esophageal cancer in vivo [31]. So authors supposed TLR-4 played its role in neoplastic progression in $\mathrm{BE}$ through facilitating COX-2 expression.

\section{The role of CAFs in the proliferation and angiogenesis of esophageal cancer}

Fibroblast growth factor (FGF) and HGF were involved in many biological activities including proliferation, differentiation, and cellular motility and morphogenesis $[32,33]$. In ESCC, they could be expressed in CAFs and promoted tumor cells proliferation via HGF/MET and
FGF/FGF receptors (FGFR) pathways. Shin et al. [6] observed that ESCC cells proliferation was boosted when they were cultured with FGF and HGF. And this process could be blocked by MET inhibitor and FGFR inhibitor. FGFR2-positive CAFs supported ESCC proliferation by creating a suitable microenvironment. A study conducted by Chunyu et al. [2] compared different expression of 126 genes between CAFs from esophageal tumor tissues and NFs, and revealed that FGFR2 was one of the most differently expressed genes. Cancer cells grew rapidly when cultured in conditioned medium from FGFR2-positive CAFs. The medium was detected to contain growth factors and transcription factors, such as FGF, transcription factor AP- $2 \alpha$ and AP-2 $\gamma$, all of which were suitable for esophageal cancer cell proliferation.

Wingless-type MMTV integration site family member 2 (Wnt2) was a member of Wnt signaling pathways which played multiple roles in regulating cellular behavior. It was revealed that Wnt2 secreted by CAFs was able to mediate tumor cell growth. Fu et al. [34] investigated tumor tissues from 51 primary ESCC patients by immunohistochemical staining and found that Wnt2 was detectable in $82.4 \%$ cases. The majority of Wnt2-positive cells were located around tumor nest. Moreover, Wnt2 and the fibroblast marker vimentin were verified to coexpress in the same cells. Thus, the authors considered Wnt2-positive cells as CAFs. It was further demonstrated that the proliferation rate of ESCC cells was higher in conditioned medium containing secreted Wnt 2 than that of control group.

Vascular endothelial growth factor (VEGF) could stimulate angiogenesis and anti-VEGF therapies were crucial in some cancer treatments [35]. Noma et al. [14] created a novel 3D model to study angiogenesis in ESCC. It was reported angiogenesis and high-level VEGF existed in the model but not in that without CAFs. To further test the role of CAFs in angiogenesis, the authors suppressed their activation by adding TGF $\beta$ inhibitors. As a result, VEGF and vascular network formation were hardly detected. In contrast, both of them were rescued when the suppression was removed. Therefore, CAFs were indispensable in angiogenesis.

Additionally, CAFs were able to accelerate EAC growth. Underwood et al. [9] respectively injected OE33 (EAC cell line), OE33+NFs and OE33+CAFs into three groups of mice. The tumor volume of the mice injected by EAC cells plus CAFs reached $500 \mathrm{~mm} 3$ earliest. The trend was verified by another EAC cell line OANC1. Another study [36] revealed that CAFs expressed VEGF to facilitate angiogenesis during tumor growth. This provided evidence for the similar involvement of CAFs in angiogenesis in ESCC and EAC. 


\section{The role of CAFs in esophageal cancer invasion and metastasis}

Invasion and metastasis were the most common causes of cancer-related death, which were related to some factors from CAFs including HGF, transforming growth factor beta-induced protein (TGF $\beta \mathrm{I})$, Wnt2, periostin and podoplanin [7-10].

HGF was an invasiveness-promoting factor in ESCC. This was supported by Grugan et al. [10] who revealed invasive epithelial cells were induced by the stimulation of CAF-derived HGF. And the process could be blocked by MET (known as the receptor of HGF) inhibitor and be enhanced by MET activation.

TGF $\beta$ I exhibited different role in cancer progression. For example, it promoted colon cancer cells metastasis and suppressed mesothelioma progression [37, 38]. In ESCC, TGF $\beta$ I was a promoter which was mainly detected in fibroblasts. Authors verified it by wound healing and invasion assays. Compared with control group, migration and invasion abilities of ESCC cells were suppressed dramatically when they were cocultured with TGF $\beta$ Idownregulated fibroblasts [8].

Epithelial-mesenchymal transition (EMT) was a process in which the epithelium-origin tumor cells lost the polarity and intercellular adhesion, and gained a propensity for migration and invasion to become mesenchymal cells. CAF-derived Wnt2 could enhance motility and invasiveness of esophageal cancer cells via EMT. Incubated in medium containing Wnt2, ESCC cells showed down-regulated epithelial markers, up-regulated mesenchymal markers and enhanced capacity to invade through extracellular matrix by up to $75 \%$ [34].

In terms of EAC cells invasiveness, Underwood et al. [9] demonstrated two EAC cell lines FLO-1 and OE33 showed a more than twofold increasing invasion in Transwell invasion assays when exposed in conditioned medium from CAFs compared with that from NFs. This trend was in accordance with results of the organic culture. They measured the average tumor invasion depth, the number and area of invading tumor islands as the parameters of cancer cells invasiveness. Strengthened invasion of FLO-1 and OE33 cells was observed in CAFscontaining gels. In order to clarify its mechanism, authors examined the CAFs and NFs conditioned medium and found more periostin in the former one. Periostin is crucial for cell adhesion and migration. Then, they respectively eliminated periostin in RNA and protein level from two groups of CAFs-conditioned medium. Neither did the medium support EAC invasion in Transwell assay nor in the organic culture. This was restored through periostin addition. In addition, authors found PI3k-Akt pathway activated by periostin was involved in the process. This supported the notion that EAC invasiveness was fueled by periostin secretion by CAFs. Except for periostin, the lymphatic endothelium marker podoplanin expressed in CAFs correlated with the EAC lymphovascular invasion and lymph node metastasis [39].

A majority of studies merely focused on ESCC or EAC alone, but there were a few studies investigating proteins in both of them, showing the histological-specific feature. For example, the stromal podoplanin and carbonic anhydrase IX (CA IX) which were associated with lymph node metastasis in EAC did not make the same impact on ESCC [39-41]. Notably, the association of CA IX with CAFs was verified in EAC rather than ESCC [41]. Further study should clarify its source in ESCC.

\section{CAFs and esophageal cancer prognosis}

As a highly lethal disease, it is significant to identify prognosis indicators of esophageal cancer, which impacts the treatment options. Besides their role in carcinogenesis, proliferation, angiogenesis, invasion and metastasis, proteins of CAFs were reported as prognosis markers for esophageal cancer patients. Studies revealed that immature CAF phenotype and CAF indicator $\alpha$-SMA was respectively related to the poor survival of ESCC and EAC patients $[9,42]$.

In ESCC patients, CD10 was expressed in CAFs. Authors found the CD10 overexpressing was correlated with decreased tumor differentiation, poor overall survival (OS) and disease free survival (DFS) [43]. Fu et al. [34] examined 51 tumor specimens from ESCC patients who received operation alone without preoperative treatment. CAFs-derived Wnt2 showed significant association with lymph node metastasis and short median survival time (Wnt2-positive ESCC vs Wnt2-negative ESCC, 16 vs. 51 months). Ozawa et al. [8] observed strong expression of TGF $\beta I$ in fibroblasts. Its expression was an independent predictor of OS. Thus, all the literatures reviewed above led to the conclusion that some productions of CAFs were a potential prognostic marker in ESCC.

In EAC patients, CAF-originated periostin also served as a survival predictor. Periostin was detected where there was high $\alpha$-SMA expression. This suggested that periostin was released from CAFs. Further study demonstrated that patients with periostin-positive in tumor had worse prognosis than those periostin-negative patients in OS (46.80 vs. 76.45 months) and DFS (47.45 vs. 80.67 months), respectively [9]. The transmembrane sialoglycoprotein podoplanin exhibited its prognostic value in EAC patients as well. Schoppmann et al. [39] investigated EAC patients who underwent operation and found that podoplanin-expressing CAFs were positively associated short OS (42 vs. 105 months) and mean DFS (42 vs. 89 months). So no matter what disease patients 
suffered, high level of these proteins predicted poor prognosis.

\section{Future clinical application of CAFs in esophageal cancer treatment}

Despite comprehensive treatment, the prognosis of esophageal cancer remains dismal. Present therapies such as chemotherapy, radiotherapy and targeted-treatment emphasized elimination cancer cells per se. However, according to the present review, carcinogenesis, cancer angiogenesis, metastasis and prognosis were all remarkably affected by stromal microenvironment. Thus, we believed blocking epithelial-fibroblasts crosstalk may be a promising therapeutic management of esophageal cancer.

Currently, studies concerning clinical CAFs-targeted treatment were limited to in vitro studies and animal models. In the study of Shin et al. [6], ESCC cells proliferation was increased when they were incubated in CAFs supernatants. This could be impaired by MET inhibitor PHA-665752 and FGFR inhibitor PD-173074. Achyut et al. [28] deleted T $\beta$ RII in fibroblasts. They found inflammation and squamous cell carcinoma developed in the forestomach of the mouse. When mouse were treated with anti-inflammation celecoxib, the tumor mass was decreased and their life expectancy increased from 28 to 49 days. The authors believed this model in forestomach could represent ESCC due to similar histology and functional behavior. Although studies on MET inhibitor, FGFR inhibitor and anti-inflammation treatment in cancer suppression were limited, they may provide a potential treatment method for further investigation.

Precisely predicting prognosis of esophageal cancer had long been a focus in clinical practice, for it may affect treatment choice. As was mentioned above, some factors from CAFs may serve as potential biomarkers for prognostic prediction, though few of them were used in clinic by far. Obstacles for its clinical use may be that the detection process is time-consuming and costly. Thus, further investigation was needed.

\section{Conclusion}

CAFs are a subtype of activated fibroblast and originate from numerous types of cells. They have pleiotropic functions in esophageal carcinogenesis, proliferation, angiogenesis, metastasis and prognosis prediction. We reviewed CAFs sources and summarized the role of CAFs-derived proteins in each step of esophageal cancer development, the details of which were shown in Table 1.

Table 1 The role cancer-associated fibroblasts play in esophageal cancer

\begin{tabular}{|c|c|c|c|c|c|}
\hline $\begin{array}{l}\text { Stages during esophageal } \\
\text { cancer development }\end{array}$ & $\begin{array}{l}\text { Functional factors } \\
\text { expressed by CAFs }\end{array}$ & $\begin{array}{l}\text { In RE/BE/ } \\
\mathrm{ESCC} / \mathrm{EAC}\end{array}$ & $\begin{array}{l}\text { Positive/negative } \\
\text { function }\end{array}$ & $\begin{array}{l}\text { Mechanism or postulated } \\
\text { mechanism }^{\mathrm{a}}\end{array}$ & References \\
\hline \multirow[t]{3}{*}{ Premalignant condition } & IL-6 & RE & + & Promoting inflammation & {$[22]$} \\
\hline & HB-EGF & $\mathrm{BE}$ & + & Promoting metaplasia & {$[5]$} \\
\hline & $\operatorname{cox} 2$ & $\mathrm{BE}$ & + & $\begin{array}{l}\text { Promoting proliferation } \\
\text { through PGE }\end{array}$ & {$[25]$} \\
\hline \multirow[t]{3}{*}{ Carcinogenesis } & TGF $\beta 1$ and $H G F$ & ESCC & + & Unknown & {$[27]$} \\
\hline & $\mathrm{T} \beta R \|$ & ESCC & - & TGF- $\beta$ signaling pathway & {$[28]$} \\
\hline & TLR-4 & EAC & + & COX-2/MAPK pathway ${ }^{a}$ & {$[29]$} \\
\hline \multirow[t]{6}{*}{ Proliferation and angiogenesis } & FGF & ESCC & + & FGF/FGFR pathways & {$[6]$} \\
\hline & HGF & ESCC & + & HGF/MET pathways & {$[6]$} \\
\hline & FGFR2 & ESCC & + & $\begin{array}{l}\text { Creating suitable environment } \\
\text { for cancer cells proliferation }\end{array}$ & {$[2]$} \\
\hline & Wnt2 & ESCC & + & $\begin{array}{l}\text { Wnt/ } \beta \text {-catenin signaling } \\
\text { pathway }\end{array}$ & {$[34]$} \\
\hline & VEGF & ESCC & + & Promoting angiogenesis & {$[14]$} \\
\hline & VEGF & EAC & + & Promoting angiogenesis & {$[36]$} \\
\hline \multirow[t]{4}{*}{ Invasion and metastasis } & HGF & ESCC & + & HGF/MET signaling pathway & {$[10]$} \\
\hline & TGF $\beta 1$ & ESCC & + & $\begin{array}{l}\text { Promoting migration and } \\
\text { invasion }\end{array}$ & {$[8]$} \\
\hline & Wnt2 & ESCC & + & Promoting EMT of cancer cells & {$[34]$} \\
\hline & Periostin & EAC & + & PI3k-Akt pathway & [9] \\
\hline
\end{tabular}

Through secreting factors, CAFs exerted an influence on esophageal cancer development

$R E$ reflux esophagitis, BE Barrett's esophagus, IL interleukin, $H B$-EGF heparin-binding EGF-like factor, (COX)-2 cyclooxygenase, TGF $\beta 1$ transforming growth factor $\beta 1$, $H G F$ hepatocyte growth factor, T $\beta R I I T G F \beta$ receptor $2, T L R-4$ toll-like receptor-4, FGF fibroblast growth factors, FGFR fibroblast growth factors receptor, Wnt2 winglesstype MMTV integration site family member 2,VEGF vascular endothelial growth factor, TGF $\beta$ I transforming growth factor beta-induced protein, $C A I X$ carbonic anhydrase IX, PGE prostaglandin E, MAPK mitogen-activated protein kinases, EMT epithelial-mesenchymal transition

a postulated mechanism 
Podoplanin and CA IX exerted different impact on ESCC and EAC, while VEGF exerted same impact. Given studies elucidating interaction between CAFs and tumor cells, many clinical investigations were conducted. It was commonly suggested factors derived from CAFs served as negative prognosis predictor. Additionally, a few explorations of preclinical treatment targeting the proteins have already been made. Though further researches were needed, we believe interpretation of CAFs in esophageal cancer will increase our knowledge of esophageal cancer and favor future clinical practice.

\begin{abstract}
Abbreviations
BMDCs: bone marrow-derived cells; BE: Barrett's esophagus; CAFs: cancerassociated fibroblasts; CA IX: carbonic anhydrase IX; CKI: cyclin-dependent kinase inhibitor; COX: cyclooxygenase; DFS: disease free survival; EMT: epithelial-mesenchymal transition; EAC: esophageal adenocarcinoma; ESCC: esophageal squamous cell carcinoma; ECM: extracellular matrix; FGF: fibroblast growth factors; FGFR: FGF receptors; IL: interleukin; HSCs: hematopoietic stem cells; HB-EGF: Heparin-binding EGF-like factor; HGF: hepatocyte growth factor; NFs: normal fibroblasts; OS: overall survival; PGE2: prostaglandins E2; SMA: smooth muscle actin; T $\beta R$ II: TGF $\beta$ receptor 2; TLR-4: toll-like receptor-4; TGF $\beta 1$ : transforming growth factor $\beta 1$; TGF $\beta$ : transforming growth factor beta; TGF $\beta$ : transforming growth factor beta-induced protein; VEGF: vascular endothelial growth factor; Wnt2: wingless-type MMTV integration site family member 2.
\end{abstract}

\section{Authors' contributions}

JFW searched the related articles and drafted the manuscript. GZ searched the related articles and revised the manuscript. JBW searched the related article and made tables and figures. XH and LW searched the related articles and revised the manuscript. YC designed the study, read and approved for publication. All authors read and approved the final manuscript.

\section{Acknowledgements}

The work was supported by Youth Science Found of Qilu Hospital of Shandong University, Science and Technology plan project of Shandong province 2012GSF11852, and National Natural Science Foundation of China 81572958.

\section{Competing interests}

The authors declare that they have no competing interests.

Received: 3 August 2015 Accepted: 17 January 2016

Published online: 29 January 2016

\section{References}

1. Pennathur A, Gibson MK, Jobe BA, Luketich JD. Oesophageal carcinoma. Lancet. 2013;381(9864):400-12. doi:10.1016/s0140-6736(12)60643-6.

2. Zhang C, Fu L, Fu J, Hu L, Yang H, Rong TH, et al. Fibroblast growth factor receptor 2-positive fibroblasts provide a suitable microenvironment for tumor development and progression in esophageal carcinoma. Clin Cancer Res. 2009;15(12):4017-27. doi:10.1158/1078-0432.ccr-08-2824.

3. Kalluri R, Zeisberg M. Fibroblasts in cancer. Nat Rev Cancer. 2006;6(5):392401. doi:10.1038/nrc1877.

4. Okawa T, Michaylira CZ, Kalabis J, Stairs DB, Nakagawa H, Andl CD, et al. The functional interplay between EGFR overexpression, hTERT activation, and p53 mutation in esophageal epithelial cells with activation of stromal fibroblasts induces tumor development, invasion, and differentiation. Genes Dev. 2007;21(21):2788-803. doi:10.1101/gad.1544507.

5. Rahman FB, Kadowaki Y, Ishihara S, Tobita H, Imaoka H, Fukuhara H, et al. Fibroblast-derived HB-EGF promotes Cdx2 expression in esophageal squamous cells. Lab Invest. 2010;90(7):1033-48. doi:10.1038/ labinvest.2010.71.
6. Saito S, Morishima K, Ui T, Hoshino H, Matsubara D, Ishikawa S, et al. The role of HGF/MET and FGF/FGFR in fibroblast-derived growth stimulation and lapatinib-resistance of esophageal squamous cell carcinoma. BMC Cancer. 2015;15:82. doi:10.1186/s12885-015-1065-8.

7. Saadi A, Shannon NB, Lao-Sirieix P, O'Donovan M, Walker E, Clemons $\mathrm{NJ}$, et al. Stromal genes discriminate preinvasive from invasive disease, predict outcome, and highlight inflammatory pathways in digestive cancers. Proc Natl Acad Sci USA. 2010;107(5):2177-82. doi:10.1073/ pnas.0909797107.

8. Ozawa D, Yokobori T, Sohda M, Sakai M, Hara K, Honjo H, et al. TGFBI expression in cancer stromal cells is associated with poor prognosis and hematogenous recurrence in esophageal squamous cell carcinoma. Ann Surg Oncol. 2014;. doi:10.1245/s10434-014-4259-4.

9. Underwood TJ, Hayden AL, Derouet M, Garcia E, Noble F, White MJ, et al. Cancer-associated fibroblasts predict poor outcome and promote periostin-dependent invasion in oesophageal adenocarcinoma. J Pathol. 2015;235(3):466-77. doi:10.1002/path.4467.

10. Grugan KD, Miller CG, Yao Y, Michaylira CZ, Ohashi S, Klein-Szanto AJ, et al. Fibroblast-secreted hepatocyte growth factor plays a functional role in esophageal squamous cell carcinoma invasion. Proc Natl Acad Sci USA. 2010;107(24):11026-31. doi:10.1073/pnas.0914295107.

11. Harper J, Sainson RC. Regulation of the anti-tumour immune response by cancer-associated fibroblasts. Semin Cancer Biol. 2014;25:69-77. doi:10.1016/j.semcancer.2013.12.005.

12. Nouraee N, Van Roosbroeck K, Vasei M, Semnani S, Samaei NM, Naghshvar F, et al. Expression, tissue distribution and function of miR-21 in esophageal squamous cell carcinoma. PLoS ONE. 2013;8(9):e73009. doi:10.1371/journal.pone.0073009.

13. Tanaka K, Miyata H, Sugimura K, Fukuda S, Kanemura T, Yamashita K, et al. miR-27 is associated with chemoresistance in esophageal cancer through transformation of normal fibroblasts to cancer-associated fibroblasts. Carcinogenesis. 2015. doi:10.1093/carcin/bgv067.

14. Noma K, Smalley KS, Lioni M, Naomoto Y, Tanaka N, El-Deiry W, et al. The essential role of fibroblasts in esophageal squamous cell carcinoma-induced angiogenesis. Gastroenterology. 2008;134(7):1981-93. doi:10.1053/j.gastro.2008.02.061.

15. Hutchinson L, Stenstrom B, Chen D, Piperdi B, Levey S, Lyle S, et al. Human Barrett's adenocarcinoma of the esophagus, associated myofibroblasts, and endothelium can arise from bone marrow-derived cells after allogeneic stem cell transplant. Stem Cells Dev. 2011;20(1):11-7. doi:10.1089/ scd.2010.0139.

16. Xouri G, Christian S. Origin and function of tumor stroma fibroblasts. Semin Cell Dev Biol. 2010;21 (1):40-6. doi:10.1016/j.semcdb.2009.11.017.

17. Ogawa M, LaRue AC, Drake CJ. Hematopoietic origin of fibroblasts/myofibroblasts: Its pathophysiologic implications. Blood. 2006;108(9):2893-6. doi:10.1182/blood-2006-04-016600.

18. Quante M, Tu SP, Tomita H, Gonda T, Wang SS, Takashi S, et al. Bone marrow-derived myofibroblasts contribute to the mesenchymal stem cell niche and promote tumor growth. Cancer Cell. 2011;19(2):257-72. doi:10.1016/j.ccr.2011.01.020.

19. Mishra PJ, Mishra PJ, Humeniuk R, Medina DJ, Alexe G, Mesirov JP, et al. Carcinoma-associated fibroblast-like differentiation of human mesenchymal stem cells. Cancer Res. 2008;68(11):4331-9. doi:10.1158/0008-5472. can-08-0943.

20. Jotzu C, Alt E, Welte G, Li J, Hennessy BT, Devarajan E, et al. Adipose tissue derived stem cells differentiate into carcinoma-associated fibroblastlike cells under the influence of tumor derived factors. Cell Oncol. 2011;34(1):55-67. doi:10.1007/s13402-011-0012-1.

21. Bailey T, Biddlestone L, Shepherd N, Barr H, Warner P, Jankowski J. Altered cadherin and catenin complexes in the Barrett's esophagus-dysplasiaadenocarcinoma sequence: correlation with disease progression and dedifferentiation. Am J Pathol. 1998;152(1):135-44.

22. Rieder F, Cheng L, Harnett KM, Chak A, Cooper GS, Isenberg G, et al. Gastroesophageal reflux disease-associated esophagitis induces endogenous cytokine production leading to motor abnormalities. Gastroenterology. 2007;132(1):154-65. doi:10.1053/j.gastro.2006.10.009.

23. Eda A, Osawa H, Satoh K, Yanaka I, Kihira K, Ishino Y, et al. Aberrant expression of CDX2 in Barrett's epithelium and inflammatory esophageal mucosa. J Gastroenterol. 2003;38(1):14-22. doi:10.1007/s005350300001.

24. Kazumori H, Ishihara S, Rumi MA, Kadowaki Y, Kinoshita Y. Bile acids directly augment caudal related homeobox gene $\mathrm{Cd} 2 \mathrm{2}$ expression in 
oesophageal keratinocytes in Barrett's epithelium. Gut. 2006;55(1):16-25. doi:10.1136/gut.2005.066209.

25. Buttar NS, Wang KK, Anderson MA, Dierkhising RA, Pacifico RJ, Krishnadath KK, et al. The effect of selective cyclooxygenase-2 inhibition in Barrett's esophagus epithelium: an in vitro study. J Natl Cancer Inst. 2002;94(6):422-9.

26. Zhao Y, Schetter AJ, Yang GB, Nguyen G, Mathe EA, Li P, et al. microRNA and inflammatory gene expression as prognostic marker for overall survival in esophageal squamous cell carcinoma. Int J Cancer. 2013;132(12):2901-9. doi:10.1002/ijc.27954.

27. Xu Z, Wang S, Wu M, Zeng W, Wang X, Dong Z. TGFbeta1 and HGF protein secretion by esophageal squamous epithelial cells and stromal fibroblasts in oesophageal carcinogenesis. Oncol Lett. 2013;6(2):401-6. doi:10.3892/ ol.2013.1409.

28. Achyut BR, Bader DA, Robles AI, Wangsa D, Harris CC, Ried T, et al. Inflammation-mediated genetic and epigenetic alterations drive cancer development in the neighboring epithelium upon stromal abrogation of TGF-beta signaling. PLoS Genet. 2013;9(2):e1003251. doi:10.1371/journal. pgen.1003251.

29. Verbeek RE, Siersema PD, Ten Kate FJ, Fluiter K, Souza RF, Vleggaar FP, et al. Toll-like receptor 4 activation in Barrett's esophagus results in a strong increase in COX-2 expression. J Gastroenterol. 2014;49(7):1121-34. doi:10.1007/s00535-013-0862-6.

30. Taddei A, Fabbroni V, Pini A, Lucarini L, Ringressi MN, Fantappie O, et al. Cyclooxygenase-2 and inflammation mediators have a crucial role in reflux-related esophageal histological changes and Barrett's esophagus. Dig Dis Sci. 2014;59(5):949-57. doi:10.1007/s10620-013-2975-4.

31. Buttar NS, Wang KK, Leontovich O, Westcott JY, Pacifico RJ, Anderson MA, et al. Chemoprevention of esophageal adenocarcinoma by COX-2 inhibitors in an animal model of Barrett's esophagus. Gastroenterology. 2002;122(4):1101-12.

32. Brooks AN, Kilgour E, Smith PD. Molecular pathways: fibroblast growth factor signaling: a new therapeutic opportunity in cancer. Clin Cancer Res. 2012;18(7):1855-62. doi:10.1158/1078-0432.ccr-11-0699.

33. Molnarfi N, Benkhoucha M, Funakoshi H, Nakamura T, Lalive PH. Hepatocyte growth factor: A regulator of inflammation and autoimmunity. Autoimmun Rev. 2015;14(4):293-303. doi:10.1016/j.autrev.2014.11.013.
34. Fu L, Zhang C, Zhang LY, Dong SS, Lu LH, Chen J, et al. Wnt2 secreted by tumour fibroblasts promotes tumour progression in oesophageal cancer by activation of the Wnt/beta-catenin signalling pathway. Gut. 2011;60(12):1635-43. doi:10.1136/gut.2011.241638.

35. Vempati P, Popel AS. Mac Gabhann F. Extracellular regulation of VEGF: isoforms, proteolysis, and vascular patterning. Cytokine Growth Factor Rev. 2014;25(1):1-19. doi:10.1016/j.cytogfr.2013.11.002.

36. Nie L, Lyros O, Medda R, Jovanovic N, Schmidt JL, Otterson MF, et al. Endothelial-mesenchymal transition in normal human esophageal endothelial cells cocultured with esophageal adenocarcinoma cells: role of IL-1 beta and TGF-beta2. Am J Physiol Cell Physiol. 2014;307(9):C859-77. doi:10.1152/ajpcell.00081.2014.

37. $\mathrm{Ma}$ C, Rong Y, Radiloff DR, Datto MB, Centeno B, Bao S, et al. Extracellular matrix protein betaig-h3/TGFBI promotes metastasis of colon cancer by enhancing cell extravasation. Genes Dev. 2008;22(3):308-21. doi:10.1101/ gad.1632008.

38. Wen G, Hong M, Li B, Liao W, Cheng SK, Hu B, et al. Transforming growth factor-beta-induced protein (TGFBI) suppresses mesothelioma progression through the Akt/mTOR pathway. Int J Oncol. 2011;39(4):1001-9. doi:10.3892/ijo.2011.1097.

39. Schoppmann SF, Jesch B, Riegler MF, Maroske F, Schwameis K, Jomrich G, et al. Podoplanin expressing cancer associated fibroblasts are associated with unfavourable prognosis in adenocarcinoma of the esophagus. Clin Exp Metastasis. 2013;30(4):441-6. doi:10.1007/s10585-012-9549-2.

40. Ma W, Wang K, Yang S, Wang J, Tan B, Bai B, et al. Clinicopathology significance of podoplanin immunoreactivity in esophageal squamous cell carcinoma. Int J Clin Exp Pathol. 2014;7(5):2361-71.

41. Jomrich G, Jesch B, Birner P, Schwameis K, Paireder M, Asari R, et al. Stromal expression of carbonic anhydrase IX in esophageal cancer. Clin Transl Oncol. 2014;16(11):966-72. doi:10.1007/s12094-014-1180-z.

42. Ha SY, Yeo SY, Xuan YH, Kim SH. The prognostic significance of cancerassociated fibroblasts in esophageal squamous cell carcinoma. PLOS ONE. 2014;9(6):e99955. doi:10.1371/journal.pone.0099955.

43. Lee KW, Sung CO, Kim JH, Kang M, Yoo HY, Kim HH, et al. CD10 expression is enhanced by Twist 1 and associated with poor prognosis in esophageal squamous cell carcinoma with facilitating tumorigenicity in vitro and in vivo. Int J Cancer. 2015;136(2):310-21. doi:10.1002/ijc.29006.

\section{Submit your next manuscript to BioMed Central and we will help you at every step:}

- We accept pre-submission inquiries

- Our selector tool helps you to find the most relevant journal

- We provide round the clock customer support

- Convenient online submission

- Thorough peer review

- Inclusion in PubMed and all major indexing services

- Maximum visibility for your research

Submit your manuscript at www.biomedcentral.com/submit

\section{() Biomed Central}

